\title{
Prognostic significance of the Controlling Nutritional Status (CONUT) score in surgically treated breast cancer patients
}

\author{
Zhang-Zan Huang", Chen-Ge Song", Jia-Jia Huang", Wen Xia, Xi-Wen Bi, Xin Hua, Zhen-Yu He*, \\ Zhong-Yu Yuan* \\ Department of Medical Oncology, Sun Yat-sen University Cancer Center, State Key Laboratory of Oncology in South China, Collaborative \\ Innovation Center for Cancer Medicine, Guangzhou, China \\ Contributions: (I) Conception and design: ZY Yuan; (II) Administrative support: ZY He; (III) Provision of study materials or patients: X Hua; (IV) \\ Collection and assembly of data: XW Bi, W Xia; (V) Data analysis and interpretation: ZZ Huang, CG Song, JJ Huang; (VI) Manuscript writing: All \\ authors; (VII) Final approval of manuscript: All authors. \\ \#These authors contributed equally to this work. \\ *These authors contributed equally to this work. \\ Correspondence to: Zhong-Yu Yuan, MD, PhD. Department of Medical Oncology, Sun Yat-Sen University Cancer Center, State Key Laboratory of Oncology \\ in South China, Collaborative Innovation Center for Cancer Medicine, 651 Dongfeng East Road, Guangzhou, China. Email: yuanzhy@sysucc.org.cn; \\ Zhen-Yu He, MD, PhD. Department of Radiotherapy, Sun Yat-sen University Cancer Center, State Key Laboratory of Oncology in South China, \\ Collaborative Innovation Center for Cancer Medicine, 651 Dongfeng East Road, Guangzhou, China. Email: hezhy@sysucc.org.cn.
}

Background: Breast cancer is one of the most common malignancy in women with high mortality rate. Given the growing evidence shows that immune-inflammatory system influences the survival of patients with cancer, we assessed the prognostic significance of the preoperative Controlling Nutritional Status (CONUT) score in patients with breast cancer who underwent surgery.

Methods: We conducted a retrospective analysis of 1,367 breast cancer patients who underwent surgery between December 2010 and October 2012. All individual preoperative serum albumin concentration, total cholesterol concentration, and total peripheral lymphocyte count were counted to calculate CONUT. Higher CONUT score is in line with worse nutritional status. The optimal cut-off of CONUT score was set at 3 to categorize the investigated patients into two groups, namely a high- or low-CONUT score group. We adopted univariate and multivariate analyses (Cox proportional hazards regression model) statistical method.

Results: Patients in the high-CONUT score group had shorter overall survival (OS) and recurrencefree survival (RFS) in comparison with those in the low-CONUT score group, 66.43 vs. 69.30 months and 54.70 vs. 59.98 months respectively (all $\mathrm{P}$ value $<0.05$ ). Univariate and multivariate analyses revealed that the CONUT score was an independent predictor of $\mathrm{OS}(\mathrm{P}=0.029$ and 0.046 , respectively) and $\mathrm{RFS}(\mathrm{P}=0.001$, $\mathrm{P}=0.013$, respectively).

Conclusions: The CONUT score was identified as an independent prognostic indicator in surgically treated breast cancer patients, indicating that, compared with the low CONUT score, a high CONUT score may lead to poorer prognosis.

Keywords: CONUT; breast cancer; surgery; survival

Submitted Feb 28, 2020. Accepted for publication Sep 03, 2020.

doi: $10.21037 / g s-20-294$

View this article at: http://dx.doi.org/10.21037/gs-20-294 


\section{Introduction}

Breast cancer is one of the most common malignancy in women with high mortality rate (1). By far, a set of canonical management of breast cancer has been constructed, including operation, neoadjuvant therapy, proper postoperative treatment and the recent hot immunotherapy $(2,3)$. According to the specific situation of each individual, clinician would choose the optimal treatments (4). Years of development, mature process has been formed in clinic. Despite that multimodal treatment has significantly decreased its mortality rate, breast cancer is still a severe disease imperiling thousands of patients health with the global occurrence is still increasing (5). At the same time, the medical model of precision medicine is increasingly playing an important role. A core principle of precision medicine is that cancer treatment aims at emphasizing the clinical and biological characteristics of the individual tumor $(6,7)$. Breast cancer is a highly heterogeneous malignant disease. Combining personalized treatment with individuals' conditions may embody the application of precision medicine in breast cancer and produce predictable results to improve prognosis.

Recently, inflammation and immunity have been the focus of research, depicting a promising future for cancer treatment (8). Likewise, several researches have revealed immune biomarkers as meaningful hallmarks of cancer $(9,10)$. Further, there have been studies demonstrating that activation of the host immune system is beneficial for improving the patients' overall survival $(11,12)$. Biomarkers such as the platelet to lymphocyte ratio (PLR), neutrophil to lymphocyte ratio (NLR), and C-reactive protein (CRP) have been verified as independent prognostic factors in various cancers, including breast cancer (13-17). Comparably, the Controlling Nutritional Status (CONUT) score is another emerging biomarker score which is calculated by three parameters: the serum albumin concentration, total cholesterol concentration, and total peripheral lymphocyte count; reflecting both the nutritional and immune context of the investigated patient (18). The CONUT score has reported positive outcome in several carcinomas, such as renal, gastric, prostate, and colorectal cancer (19-22). An interesting appearance was observed that patients in high CONUT group had longer survival than those in low group. Therefore, we presumed that the CONUT score could have similar impact in breast cancer and could be implemented in daily clinical practices to monitor the patient's disease condition, assessing possibility of early disease progression and to guide timely therapeutic intervention. It is an extension of precision medicine in clinical application.

In this study, we investigated the clinical applicability of the CONUT score as a new, accurate and sensitive prognostic biomarker in breast cancer and its significance to overall survival and recurrence-free survival.

We present the following article in accordance with the STROBE reporting checklist (available at http://dx.doi. org/10.21037/gs-20-294).

\section{Methods}

\section{Patients}

There were 1987 patients received curative surgery from December 2010 to October 2012 at the Sun Yat-sen University Cancer Center (SYSUCC; Guangzhou, China). The inclusion criteria were as follows: (I) breast cancer patients; (II) patients received surgery. The exclusion criteria were as follows: (I) synchronal malignancies; (II) ductal carcinoma in situ; (III) incomplete blood sample data and missing visit due to various reasons. Finally, we retrospectively retrieved the data of 1,367 breast cancer patients (see Figure 1).

\section{Ethical statement}

The study was conducted in accordance with the Declaration of Helsinki (as revised in 2013). The study was approved by the Research Ethics Committee of SYSUCC (B2020-215-01) and individual consent for this retrospective analysis was waived.

\section{Sample collection and classification}

All patients' information was retrieved from the Sun Yatsen University Cancer Center (SYSUCC) medical records. Blood samples were collected and measured within 1 week before surgery. The CONUT score was calculated using the preoperative data on serum albumin concentration, total cholesterol concentration, and total peripheral lymphocyte count. The three parameters scores were grouped into four levels based on their concentrations (Table 1). The cut-off value for the CONUT was defined as 3, according to previous research on renal, gastric, and prostate cancer (19-21). The age parameter was classified into two groups based on its median value (48 years old). The 


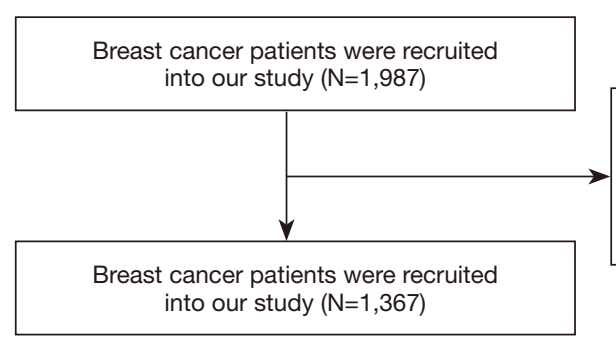

Records exclusion

Due to synchronal malignancies $(\mathrm{N}=4)$;

Due to ductal carcinoma in situ( $\mathrm{N}=66)$;

Due to incomplete blood sample data $(\mathrm{N}=252)$;

Due to lost visit ( $\mathrm{N}=298$ ).

Figure 1 Flow diagram of study selection.

Table 1 Controlling nutritional status index score: assessment of malnutritional state (20)

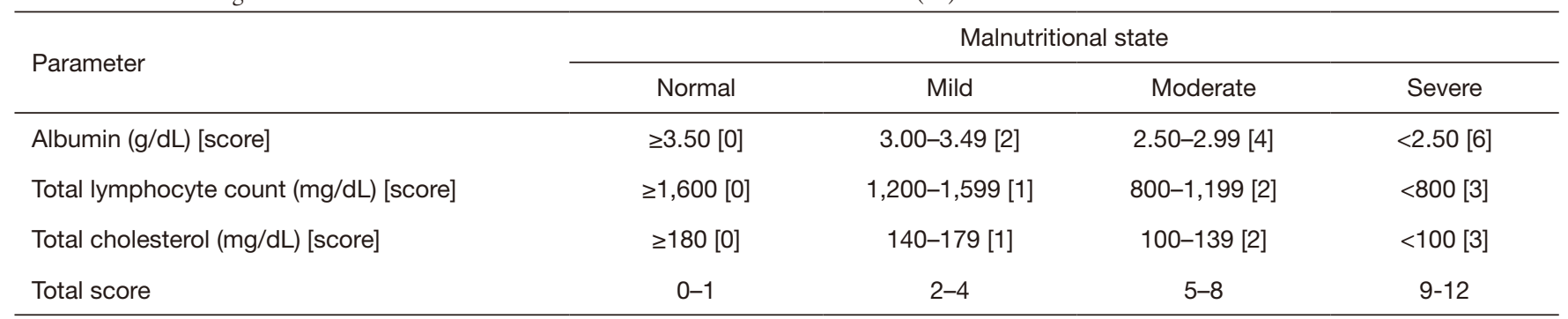

tumors were staged according to the $7^{\text {th }}$ AJCC (American Joint Committee on Cancer) TNM staging system. The expression of estrogen receptor (ER), progesterone receptor (PR), human epidermal growth factor receptor-2 (HER2) and Ki-67 were defined by the St Gallen criteria (23). We identified ER and PR positive if there were at least $1 \%$ positive heterologous tumor cell nuclei in the sample evaluated by immunohistochemistry (IHC). HER-2 status was assessed using a semiquantitative score $(0-3+)$. Patients with 2+ IHC staining for HER2 underwent fluorescence in-situ hybridization (Fish) to confirm HER2 positivity or negativity. Ki67 was stratified into two group and the cutoff was $14 \%$. The expression of tumor markers (CEA, CA153) was considered as positive if they were beyond normal range.

\section{Follow-up}

All patients were followed-up by outpatient examination or telephonic interviews. The last follow-up time was 27 September, 2019. Overall survival (OS) time was defined as the period from surgery to death from various causes or to the last follow-up date. Recurrence-free survival (RFS) was defined as the time from the date of surgery to the date of the first recurrence, death from any cause or last follow-up.

\section{Statistical analysis}

All analyses were conducted using the SPSS software version 23.0 (IBM Corp., Armonk, NY, USA) and GraphPad Prism version 6.0 (GraphPad, La Jolla, CA, USA). Survival curves were calculated using the KaplanMeier method and compared using the log-rank test. Univariate and multivariate analyses were performed using the Cox proportional hazards model. Two-tailed $\mathrm{P}$ values $<0.05$ were considered as statistically significant.

\section{Results}

\section{The optimal cut-off value for the CONUT score}

Based on their preoperative data, we calculated all patients scores and divided them into four levels, namely, normal [0-1], mild [2-4], moderate [5-8], and severe [9-12] (Table 1). According to previous study, the best CONUT cutoff was found to be 3 . Therefore, the investigated 1,367 breast cancer patients were classified into a low CONUT score group ( $<3)$ or a high CONUT score $(\geq 3)$ group.

\section{Patient characteristics and Relationships between CONUT score with clinicopathological features}

The median follow-up time was 5.86 years (range, $0.02-$ 
8.82). The clinicopathological features of the entire study cohort and the relationships between the CONUT score and patient characteristics are shown in Table 2. Based on prespecified criteria, 308 (22.5\%) and 1,059 (77.5\%) were categorized into a high- and low-CONUT score group, respectively. The CONUT score was significantly associated with age $(\mathrm{P}<0.001)$, pTNM stage $(\mathrm{P}=0.027), \mathrm{Ki}-$ $67(\mathrm{P}=0.034)$.

\section{Survival outcomes prognostication based on the CONUT score}

Figure 2 demonstrate significant survival differences between the high- and low-CONUT breast cancer groups. The OS and RFS for patients in the high- and low-CONUT groups were 64.43 and 69.30 months $(\mathrm{P}=0.026)$ and 54.70 and 56.98 months $(\mathrm{P}=0.011)$, respectively. Multivariate analyses showed that $\mathrm{N}$ stage $(\mathrm{P}<0.001), \mathrm{M}$ stage $(\mathrm{P}<0.001)$ and CONUT $(\mathrm{P}=0.046)$ matter were independent factor for OS (Table 3), while $\mathrm{N}$ stage $(\mathrm{P}=0.003), M$ stage $(\mathrm{P}<0.001)$, and CONUT $(\mathrm{P}=0.013)$ matter were independent factor for RFS (Table 4).

\section{Discussion}

Findings from the present study showed that patients with

Table 2 Clinicopathologic characteristics of the patients

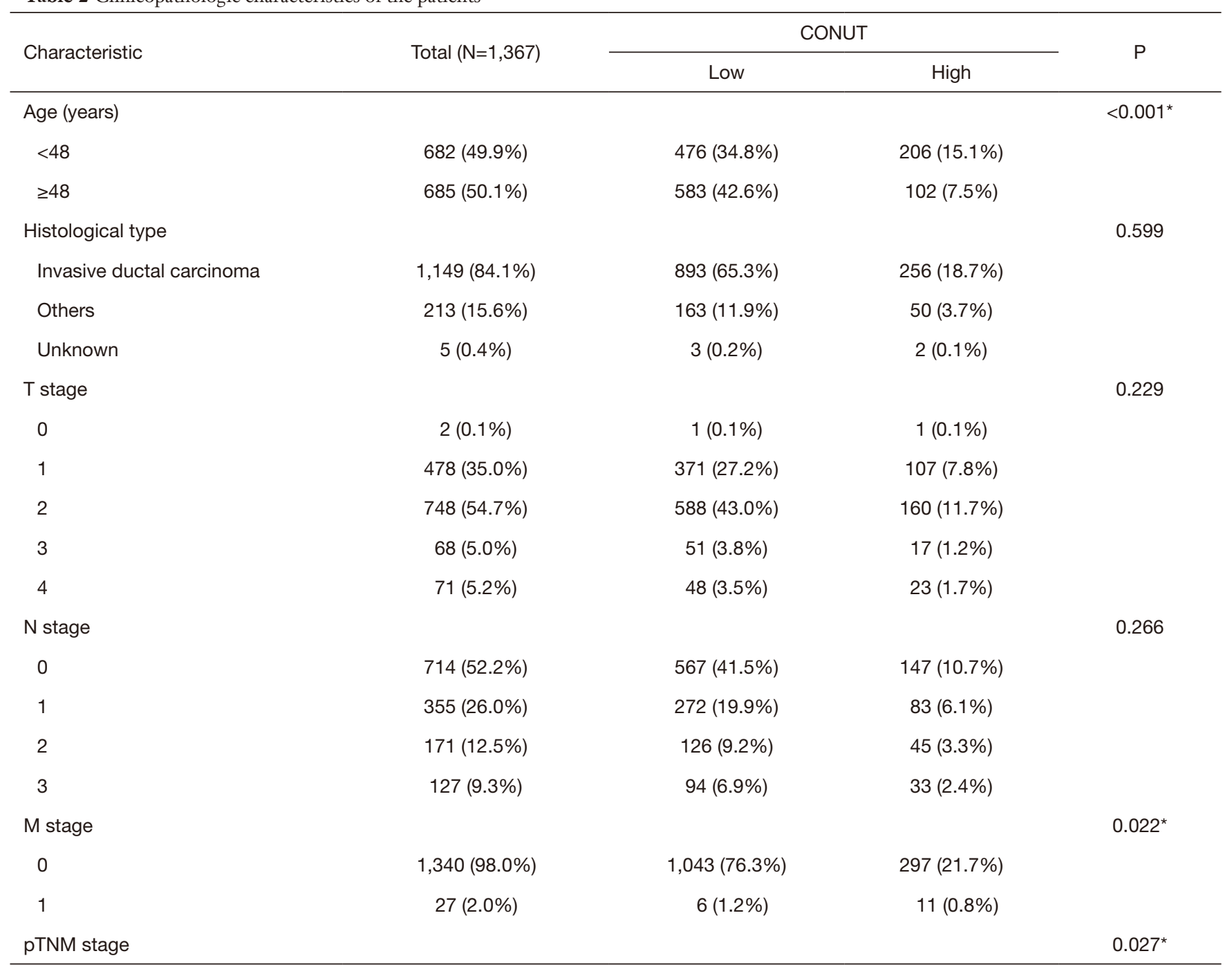

Table 2 (continued) 
Table 2 (continued)

\begin{tabular}{|c|c|c|c|c|}
\hline Characteristic & Total $(\mathrm{N}=1,367)$ & \multicolumn{2}{|c|}{ CONUT } & $\mathrm{P}$ \\
\hline I & $324(23.7 \%)$ & 255 (18.7\%) & $69(5.0 \%)$ & \\
\hline II & $694(50.8 \%)$ & $550(40.3 \%)$ & $144(10.5 \%)$ & \\
\hline III & $322(23.6 \%)$ & $238(17.5 \%)$ & $84(6.1 \%)$ & \\
\hline ER & & & & 0.276 \\
\hline Negative & $427(31.2 \%)$ & $323(23.6 \%)$ & 104 (7.6\%) & \\
\hline Positive & $940(68.8 \%)$ & 736 (53.9\%) & 204 (14.9\%) & \\
\hline PR & & & & 0.823 \\
\hline HER2 & & & & 0.986 \\
\hline Negative & $973(71.2 \%)$ & $754(55.2 \%)$ & $219(16.0 \%)$ & \\
\hline Positive & $386(28.2 \%)$ & 299 (21.9\%) & 87 (6.4\%) & \\
\hline Unknown & $8(0.6 \%)$ & $6(0.4 \%)$ & $2(0.1 \%)$ & \\
\hline Ki-67 & & & & $0.034^{\star}$ \\
\hline$\leq 14 \%$ & $498(36.4 \%)$ & $370(27.1 \%)$ & 128 (9.4\%) & \\
\hline$>14 \%$ & $869(63.6 \%)$ & 698 (50.4\%) & $180(13.2 \%)$ & \\
\hline Molecular subtype & & & & 0.353 \\
\hline Unknown & $5(0.4 \%)$ & $3(0.2 \%)$ & $2(0.1 \%)$ & \\
\hline Preoperative CEA & & & & 0.850 \\
\hline Negative & $1,190(87.1 \%)$ & $924(67.6 \%)$ & 267 (19.5\%) & \\
\hline Positive & $119(8.7 \%)$ & $93(6.8 \%)$ & $26(1.9 \%)$ & \\
\hline Unknown & $57(4.2 \%)$ & $42(3.1 \%)$ & $15(1.1 \%)$ & \\
\hline Preoperative CA153 & & & & 0.255 \\
\hline Negative & $1,150(84.1 \%)$ & $900(65.8 \%)$ & $250(18.3 \%)$ & \\
\hline Positive & $161(12.3 \%)$ & 117 (8.6\%) & $44(3.2 \%)$ & \\
\hline Unknown & $56(4.1 \%)$ & $42(3.1 \%)$ & $14(1.0 \%)$ & \\
\hline
\end{tabular}

${ }^{*} \mathrm{P}<0.05$. ER, estrogen receptor; PR, progesterone receptor; HER2, human epidermal growth factor receptor-2; CEA, carcinoembryonic antigen; CA153, cancer antigen 153. 


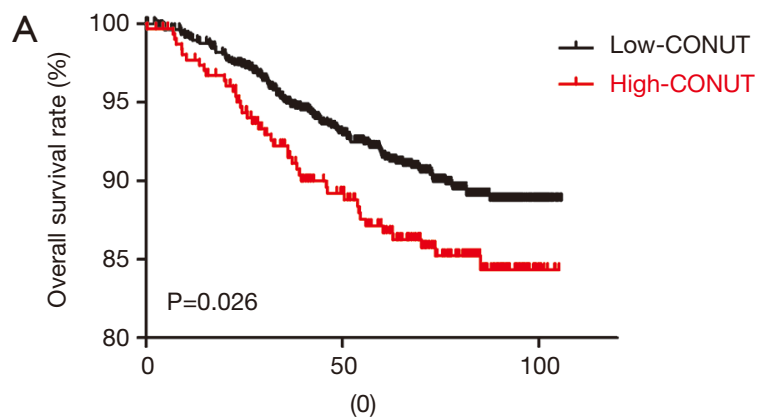

B

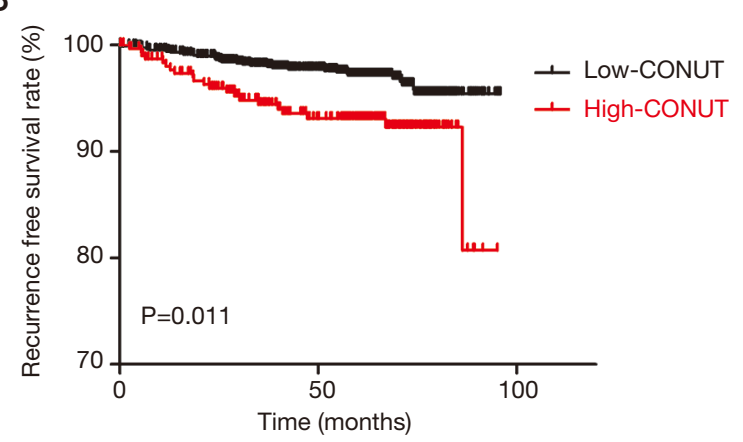

Figure 2 Kaplan-Meier for the stratified CONUT score groups in association to: (A) overall survival; (B) recurrence-free survival; and (C) distant metastatic-free survival.
high-CONUT score had poorer OS and RFS than those who with low-CONUT score and that the CONUT score was an independent prognostic factor for OS and RFS in surgically treated breast cancer patients; demonstrating that a high-CONUT score might be strongly associated with tumor progression and shorter survival.

Precision medicine request individualization $(24,25)$ and will be modern medical trend (26). Immune-inflammatory system plays critical role in many physiological activity [such as wound healing (27), infection offence (28), vaccine (29)] and malignant cancers [perhaps affects tumor microenvironment (30)], and received fanatical attention. As we all known, nutritional status is thought to be associated with prognosis on oncology (31). For those reasons, it would be a proper biomarker which can reflect subjects' immune-inflammatory system and nutritional status, moreover, stay consistent with precision medicine.

Dozens of studies about immune-inflammatory system have been reported with various cancer prognosis, including breast cancer. However, there is no indicator that owns wide applicability. CONUT is a complex score and was first introduced as an efficient tool for early detection and continuous control of hospital undernutrition $(18,32)$.

Table 3 Univariate and multivariate analyses of overall survival

\begin{tabular}{|c|c|c|c|c|}
\hline \multirow{2}{*}{ Characteristic } & \multicolumn{2}{|c|}{ Univariate analysis } & \multicolumn{2}{|c|}{ Multivariate Cox regression analysis } \\
\hline & Hazard ratio $(95 \% \mathrm{Cl})$ & $P$ & Hazard ratio $(95 \% \mathrm{Cl})$ & $P$ \\
\hline Age & $1.135(0.813-1.583)$ & 0.457 & & \\
\hline Histological type & $2.171(1.173-4.020)$ & $0.014^{\star}$ & $1.756(0.942-3.272)$ & 0.076 \\
\hline T stage & $1.864(1.559-2.227)$ & $<0.001^{*}$ & $1.149(0.918-1.437)$ & 0.226 \\
\hline N stage & $2.294(1.981-2.655)$ & $<0.001^{*}$ & $2.104(1.713-2.394)$ & $<0.001^{*}$ \\
\hline M stage & $7.983(4.499-14.166)$ & $<0.001^{*}$ & $4.221(2.275-7.834)$ & $<0.001^{*}$ \\
\hline pTNM stage & $3.574(2.833-4.509)$ & $<0.001^{*}$ & & \\
\hline ER & $0.598(0.426-0.838)$ & $0.003^{*}$ & $0.764(0.457-1.279)$ & 0.306 \\
\hline PR & $0.543(0.389-0.757)$ & $<0.001^{*}$ & $0.813(0.491-1.346)$ & 0.421 \\
\hline HER2 & $1.795(1.281-2.516)$ & $0.001^{*}$ & $1.274(0.878-1.848)$ & 0.203 \\
\hline Ki-67 & $2.116(1.413-3.169)$ & $<0.001^{*}$ & $1.515(0.990-2.320)$ & 0.056 \\
\hline Molecular subtype & $1.224(1.093-1.370)$ & $<0.001^{*}$ & & \\
\hline CEA & $3.157(2.093-4.762)$ & $<0.001^{*}$ & $1.341(0.841-2.137)$ & 0.218 \\
\hline CA153 & $1.901(1.247-2.899)$ & $0.003^{*}$ & $0.865(0.539-1.390)$ & 0.550 \\
\hline CONUT & $1.502(1.043-2.162)$ & $0.029^{*}$ & $1.465(1.007-2.132)$ & $0.046^{*}$ \\
\hline
\end{tabular}

${ }^{*} \mathrm{P}<0.05$. ER, estrogen receptor; $\mathrm{PR}$, progesterone receptor; HER2, human epidermal growth factor receptor-2; CEA, carcinoembryonic antigen; CA153, cancer antigen 153. 
Table 4 Univariate and multivariate analyses of overall survival and recurrence-free survival

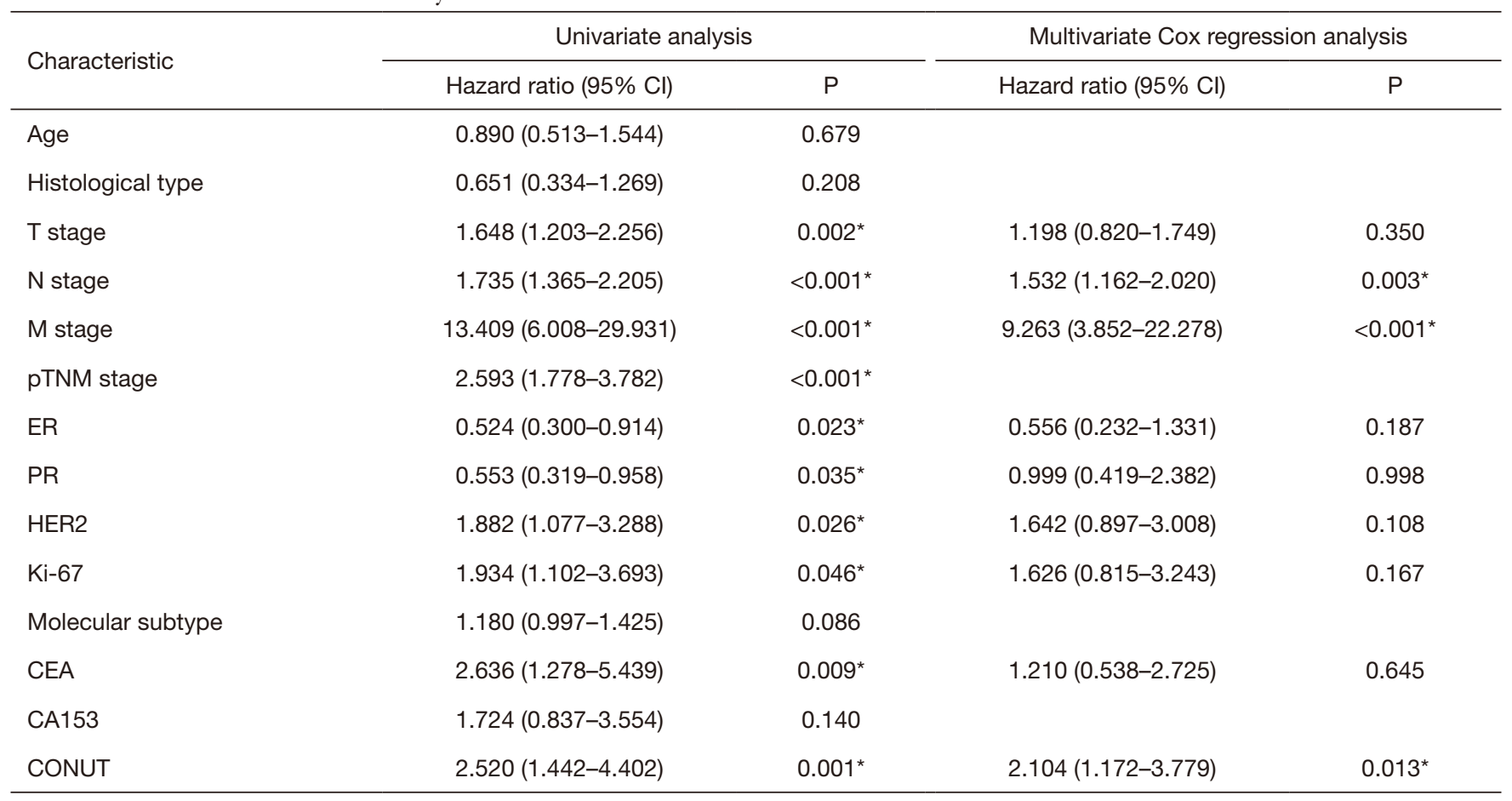

${ }^{\star} \mathrm{P}<0.05$. ER, estrogen receptor; PR, progesterone receptor; HER2, human epidermal growth factor receptor-2; CEA, carcinoembryonic antigen; CA153, cancer antigen 153.

The CONUT score could provide a more comprehensive assessment in patient nutritional and immune status. According to united scoring method, CONUT score is divided into four subunits, $0-1$ for normal, $2-4$ for mild, $5-8$ for moderate, 9-12 for severe. CONUT has been found in many studies to have substantial prognostic value for various types of cancers. In the subsequent retrospective analysis of 368 gastric cancer cases, Noriyuki Hirahara demonstrated the prognostic significance of CONUT in gastric cancer after curative gastrectomy. They also conducted a propensity score-matched analysis (PSM) in order to explore the significance. They reported that the CONUT is an objective, non-invasive, and readily available prognostic biomarker (20). In another one smaller cohort of 94 patients with oligometastatic prostate cancer, Zhang attested the relationship between high CONUT and poor PSA progression-free survival time (21). There are similar studies have been conformed in renal cancer and colorectal cancer $(22,33)$. Thus, CONUT is exactly indicator which meets current needs. Though underlying mechanism is still unclear, no study has confirmed its value in breast cancer. We therefore assumed the CONUT score will be helpful in identifying high-risk patients timely, and in providing reasonable therapy after surgery.

To our best effort, we explore the relationship between CONUT and clinicopathologic characteristics. We observed that in our study, there are $1,149(84.1 \%)$ patients were Invasive ductal carcinoma. There are 324 (23.7\%), 694 (50.8\%), $322(23.6 \%)$ and $27(2.0 \%)$ patients were clinical stage $1,2,3$ and 4 respectively. At the same time, there are $353(25.8 \%)$ patients for Luminal A, 404 (29.6\%) for Luminal B/HER2-, 202 (14.8\%) for Luminal B/HER2+, 185(13.5\%) for HER2 Enriched and 217 (15.9\%) for Triple Negative. As we all know, TNM stage is pivotal prognostic indicator. In Table 2, we noticed that TNM stage was related to CONUT $(\mathrm{P}=0.027)$. The relationship between chronic inflammation and cancer development had been studied. And some had validated chronic inflammation participated in invasion and metastasis of cancer $(34,35)$. Our results provided more robust inflammatory responses in response to more aggressive tumors and higher tumor burdens. Given the importance of TNM stage and distant metastasis in predicting breast cancer patient outcomes, our finding suggested that CONUT might function as a prognostic breast cancer biomarker.

China is a populous country with more than 1.6 million 
people being diagnosed and 1.2 million people dying of the disease each year (36). Breast cancer mortality varies from worldwide. In several reports about the mortality of China and worldwide, we observed that China held a higher mortality than worldwide, $69.5 / 100,000$ vs. $12.9 / 100,000$ (Segi Standard Population) $(37,38)$. Apart from some aligned with known risk factors for women, there are various reasons for this. The economic status is the key. Dozens of patients cannot afford cost of treatment; therefore, it is possible to quit imperative treatment. China is largest developing country in the world and a vast country, the mortality varies between urban and rural area (39). In rural area, due to the lack of popularization, many patients do not pay enough attention to the occurrence of disease, resulting in the late stage at first diagnosis. And some patients choose traditional Chinese medicine instead of going to professional tumor hospital.

Results of this study paves the way for deeper investigation about immune-inflammatory-nutritional indicators in breast cancer. Our study initially explored the relationship between CONUT and the prognosis of breast cancer patients. We found that patients with highCONUT score had shorter OS and RFS than those with low-CONUT score. CONUT is an independent predictor in breast cancer. Our results provided another perspective about precision medicine in breast cancer. Specific treatment is not only based on TNM stage, pathological type, different subtype, but also on host basic health status. Poor basic health status is a hazard for host who maybe more vulnerable about progression. Those patients could benefit from a preoperative nutritional intervention. A more intensive attention about nutritional status will be helpful in monitor disease changes.

Despite our meaningful findings, our limitations were obvious. First, our study is a retrospective analysis of a single-center design and could cause selection bias. Second, we focused on preoperative health status and failed to renew data during the whole process. Third, the underlying mechanism is unclear. Fourth, we recruited breast cancer patients are from china and we did not procedure a comparison with other countries and other races. Therefore, lots of efforts are required in order to elucidate the molecular mechanisms.

In summary, we identified the CONUT score as an independent prognostic indicator for OS and RFS in breast cancer patients after curative surgery. Patients with high CONUT score would have a greater risk of poorer survival as compared to those a low CONUT score.

\section{Acknowledgments}

Funding: None.

\section{Footnote}

Reporting Checklist: The authors have completed the STROBE reporting checklist. Available at http://dx.doi. org/10.21037/gs-20-294

Data Sharing Statement: Available at http://dx.doi. org/10.21037/gs-20-294

Peer Review File: Available at http://dx.doi.org/10.21037/gs20-294

Conflicts of Interest: All authors have completed the ICMJE uniform disclosure form (available at http://dx.doi. org/10.21037/gs-20-294). The authors have no conflicts of interest to declare.

Etbical Statement: The authors are accountable for all aspects of the work in ensuring that questions related to the accuracy or integrity of any part of the work are appropriately investigated and resolved. The study was conducted in accordance with the Declaration of Helsinki (as revised in 2013). The study was approved by the Research Ethics Committee of SYSUCC (B2020-215-01) and individual consent for this retrospective analysis was waived.

Open Access Statement: This is an Open Access article distributed in accordance with the Creative Commons Attribution-NonCommercial-NoDerivs 4.0 International License (CC BY-NC-ND 4.0), which permits the noncommercial replication and distribution of the article with the strict proviso that no changes or edits are made and the original work is properly cited (including links to both the formal publication through the relevant DOI and the license). See: https://creativecommons.org/licenses/by-nc-nd/4.0/.

\section{References}

1. Ginsburg O, Bray F, Coleman MP, et al. The global burden of women's cancers: a grand challenge in global health. Lancet 2017;389:847-60.

2. McDonald ES, Clark AS, Tchou J, et al. Clinical Diagnosis and Management of Breast Cancer. J Nucl Med 2016;57 Suppl 1:9S-16S. 
3. Emens LA. Breast Cancer Immunotherapy: Facts and Hopes. Clin Cancer Res 2018;24:511-20.

4. Harbeck N, Gnant M. Breast cancer. Lancet 2017;389:1134-50.

5. DeSantis CE, Ma J, Goding Sauer A, et al. Breast cancer statistics, 2017, racial disparity in mortality by state. CA Cancer J Clin 2017;67:439-48.

6. Tursz T, Andre F, Lazar V, et al. Implications of personalized medicine--perspective from a cancer center. Nat Rev Clin Oncol 2011;8:177-83.

7. Scott JG, Berglund A, Schell MJ, et al. A genomebased model for adjusting radiotherapy dose (GARD): a retrospective, cohort-based study. Lancet Oncol 2017;18:202-11.

8. Andrejeva G, Rathmell JC. Similarities and Distinctions of Cancer and Immune Metabolism in Inflammation and Tumors. Cell Metab 2017;26:49-70.

9. Hanahan D, Weinberg RA. Hallmarks of cancer: the next generation. Cell 2011;144:646-74.

10. Grivennikov SI, Greten FR, Karin M. Immunity, inflammation, and cancer. Cell 2010;140:883-99.

11. Savas P, Salgado R, Denkert C, et al. Clinical relevance of host immunity in breast cancer: from TILs to the clinic. Nat Rev Clin Oncol 2016;13:228-41.

12. Hodi FS, Ballinger M, Lyons B, et al. ImmuneModified Response Evaluation Criteria In Solid Tumors (imRECIST): Refining Guidelines to Assess the Clinical Benefit of Cancer Immunotherapy. J Clin Oncol 2018;36:850-8.

13. Ethier JL, Desautels D, Templeton A, et al. Prognostic role of neutrophil-to-lymphocyte ratio in breast cancer: a systematic review and meta-analysis. Breast Cancer Res 2017;19:2.

14. Graziano V, Grassadonia A, Iezzi L, et al. Combination of peripheral neutrophil-to-lymphocyte ratio and plateletto-lymphocyte ratio is predictive of pathological complete response after neoadjuvant chemotherapy in breast cancer patients. Breast 2019;44:33-8.

15. Wang J, Liu Y, Mi X, et al. The prognostic value of prognostic nutritional index (PNI) and neutrophil to lymphocyte ratio (NLR) for advanced non-small cell lung cancer treated with platinum-based chemotherapeutics. Ann Palliat Med 2020;9:967-78.

16. Mungan İ, Dicle CB, Bektas S, et al. Does the preoperative platelet-to-lymphocyte ratio and neutrophil-tolymphocyte ratio predict morbidity after gastrectomy for gastric cancer? Mil Med Res 2020;7:9.

17. Kurokawa Y, Yamashita K, Kawabata R, et al. Prognostic value of postoperative C-reactive protein elevation versus complication occurrence: a multicenter validation study. Gastric Cancer 2020;23:937-43.

18. Ignacio de Ulíbarri J, Gonzalez-Madrono A, de Villar NG, et al. CONUT: a tool for controlling nutritional status. First validation in a hospital population. Nutr Hosp 2005;20:38-45.

19. Song $\mathrm{H}, \mathrm{Xu} \mathrm{B}$, Luo C, et al. The prognostic value of preoperative controlling nutritional status score in nonmetastatic renal cell carcinoma treated with surgery: a retrospective single-institution study. Cancer Manag Res 2019;11:7567-75.

20. Hirahara N, Tajima Y, Fujii Y, et al. Controlling Nutritional Status (CONUT) as a prognostic immunonutritional biomarker for gastric cancer after curative gastrectomy: a propensity score-matched analysis. Surg Endosc 2019;33:4143-52.

21. Zhang $\mathrm{W}, \mathrm{Wu} \mathrm{Y}, \mathrm{Zhang} \mathrm{Z}$, et al. Controlling Nutritional Status score: A new prognostic indicator for patients with oligometastatic prostate cancer. Curr Probl Cancer 2019;43:461-70.

22. Yang C, Wei C, Wang S, et al. Combined Features Based on Preoperative Controlling Nutritional Status Score and Circulating Tumour Cell Status Predict Prognosis for Colorectal Cancer Patients Treated with Curative Resection. Int J Biol Sci 2019;15:1325-35.

23. Goldhirsch A, Wood WC, Coates AS, et al. Strategies for subtypes--dealing with the diversity of breast cancer: highlights of the St. Gallen International Expert Consensus on the Primary Therapy of Early Breast Cancer 2011. Ann Oncol 2011;22:1736-47.

24. Beckmann JS, Lew D. Reconciling evidence-based medicine and precision medicine in the era of big data: challenges and opportunities. Genome Med 2016;8:134.

25. Sankar PL, Parker LS. The Precision Medicine Initiative's All of Us Research Program: an agenda for research on its ethical, legal, and social issues. Genet Med 2017;19:743-50.

26. Helmy KY, Patel SA, Nahas GR, et al. Cancer immunotherapy: accomplishments to date and future promise. Ther Deliv 2013;4:1307-20.

27. Portou MJ, Baker D, Abraham D, et al. The innate immune system, toll-like receptors and dermal wound healing: A review. Vascul Pharmacol 2015;71:31-6.

28. Netea MG, Joosten LA, Latz E, et al. Trained immunity: A program of innate immune memory in health and disease. Science 2016;352:aaf1098.

29. Heppner HJ, Leischker A, Wutzler P, et al. [Vaccination in 
advanced age]. Internist (Berl) 2018;59:205-12.

30. Gajewski TF, Schreiber H, Fu YX. Innate and adaptive immune cells in the tumor microenvironment. Nat Immunol 2013;14:1014-22.

31. Mantzorou M, Koutelidakis A, Theocharis S, et al. Clinical Value of Nutritional Status in Cancer: What is its Impact and how it Affects Disease Progression and Prognosis? Nutr Cancer 2017;69:1151-76.

32. Krishnasamy K, Li Yoong T, Mei Chan C, et al. Identifying Malnutrition: Nutritional Status in Newly Diagnosed Patients With Cancer. Clin J Oncol Nurs 2017;21:E23-9.

33. Kang HW, Seo SP, Kim WT, et al. Prognostic Impact of Nutritional Status Assessed by the Controlling Nutritional Status (CONUT) Score in Patients with Surgically Treated Renal Cell Carcinoma. Nutr Cancer

Cite this article as: Huang ZZ, Song CG, Huang JJ, Xia W, Bi XW, Hua X, He ZY, Yuan ZY. Prognostic significance of the Controlling Nutritional Status (CONUT) score in surgically treated breast cancer patients. Gland Surg 2020;9(5):1370-1379. doi: 10.21037 /gs-20-294
2018;70:886-94.

34. Coffelt SB, de Visser KE. Cancer: Inflammation lights the way to metastasis. Nature 2014;507:48-9.

35. Coussens LM, Werb Z. Inflammation and cancer. Nature 2002;420:860-7.

36. Fan L, Strasser-Weippl K, Li JJ, et al. Breast cancer in China. Lancet Oncol 2014;15:e279-89.

37. Chen W, Zheng R, Baade PD, et al. Cancer statistics in China, 2015. CA Cancer J Clin 2016;66:115-32.

38. Ferlay J, Soerjomataram I, Dikshit R, et al. Cancer incidence and mortality worldwide: sources, methods and major patterns in GLOBOCAN 2012. Int J Cancer 2015;136:E359-86.

39. Bragg F, Holmes MV, Iona A, et al. Association Between Diabetes and Cause-Specific Mortality in Rural and Urban Areas of China. JAMA 2017;317:280-9. 